\title{
Dos usos de los modelos de optimalidad en las explicaciones por selección natural*
}

Two Uses of Optimality Models in Natural Selection Explanations

Santiago Ginnobili ${ }^{\dagger}$

Ariel Roffe $e^{\ddagger \mathcal{S}}$

\begin{abstract}
Resumen
El objetivo de este trabajo consiste en analizar las relaciones entre los modelos de optimalidad y la selección natural. Defenderemos que esas relaciones pueden dividirse en dos tipos, en tanto hay dos tipos de explicaciones seleccionistas, que llamaremos "históricas" y "ahistóricas". Las explicaciones históricas revelan como una población dada adquiere un rasgo que es adaptativo en ese ambiente e involucran muchas generaciones, variación, etc. Las explicaciones ahistóricas, explican por qué, en determinado momento, ciertos tipos de organismos tienen un mayor éxito reproductivo que otros. Mostraremos que los modelos de optimalidad pueden jugar un rol en la determinación del explanandum de las explicaciones histórica seleccionistas, esto es, que ayudan a reconocer qué rasgos son adaptativos. Por otra parte, mostramos que los modelos de optimalidad nos permiten determinar a veces la parte del explanans de las explicaciones ahistóricas (particularmente, el concepto de fitness).
\end{abstract}

Palabras clave: optimalidad - selección natural - fitness - teoría del forrajeo óptimo

\begin{abstract}
The goal of this paper is to analyze the relations between optimality models and natural selection. We contend that these relationships can be divided into two kinds, as there are two kinds of natural selection explanations, which we call "historical" and "ahistorical". Historical explanations reveal how a given population acquires a trait which is adaptive in its environment, and involves many generations, variations, etc. Ahistorical ones, explain why, at a given moment, certain kinds of organisms have a greater reproductive success than others. We show that optimality models can play a role in determining the explanandum of historical selectionist explanations, that is, they help us to recognize which traits are adaptive. And, on the other hand, that optimality models sometimes allow us to determine part of the explanans of ahistorical explanations (particularly, the concept of fitness).
\end{abstract}

Keywords: optimality - natural selection - fitness - optimal foraging theory

\footnotetext{
* Recibido: 30 de Marzo de 2016. Aceptado con revisiones: 5 de Diciembre de 2016.

+ Centro de Estudios de Filosofía e Historia de la Ciencia (CEFHIC), Universidad Nacional de Quilmes (UNQ)/CONICET/Universidad de Buenos Aires. Para contactar al autor, por favor, escribir a: santi75@gmail.com.

* Centro de Estudios de Filosofia e Historia de la Ciencia (CEFHIC), Universidad Nacional de Quilmes (UNQ)/CONICET/Universidad de Buenos Aires. Para contactar al autor, por favor, escribir a: ariroffe@hotmail.com.

$\$$ Esta investigación fue financiada por los siguientes proyectos: PICT-2012-2662, PICT-2014-1741 (ANPCyT, Argentina), PIP N $112-$ 201101-01135 (CONICET, Argentina) y 32/15 255 (UNTREF, Argentina).

Metatheoria 8(1)(2017): 43-55. ISSN 1853-2322.

(c) Editorial de la Universidad Nacional de Tres de Febrero. Publicado en la República Argentina.
} 


\section{Introducción}

Los estudios de optimalidad tienen una larga tradición en la historia de la biología. Por ejemplo, tanto en la biología aristotélica como en la teología natural se explicaba la presencia de un rasgo apelando al hecho de que estaba óptimamente diseñado para satisfacer su telos o su función (Blanco 2008, Henry 2013). Sin embargo, es en los años 60' y 70' del siglo XX en los que el enfoque contemporáneo de este tipo de estudios se desarrolla, vía los llamados "modelos de optimalidad". En este enfoque, y a diferencia de los anteriores, los modelos de optimalidad aparecen conceptualmente relacionados con la selección natural, de algún modo. Tanto en la literatura filosófica como en la biológica, ha habido fuertes desacuerdos con respecto a la naturaleza precisa de esta relación y, correspondientemente, acerca de la legitimidad del enfoque de optimalidad como un todo.

Por ejemplo, algunas acusaciones consisten en señalar que los modelos de optimalidad presuponen el adaptacionismo, y que en consecuencia son infalsables e inútiles (Gould \& Lewontin 1979, Gray 1987, Pierce \& Ollason 1987). Por otra parte, ha sido argüido recientemente que los modelos de optimalidad reflejan sólo las dinámicas selectivas -no las dinámicas evolutivas como un todo(Potochnik 2009); y más débilmente, que no reflejan ninguna dinámica en absoluto, o bien porque dan explicaciones de equilibrio de hacia dónde la población será conducida por la selección en el largo plazo, o bien porque meramente permiten identificar factores ambientales que podrían ser relevantes a los procesos selectivos (Bolduc \& Cézilly 2012).Por otra parte, los filósofos que defienden la interpretación propensivista del fitness sostienen que los modelos de optimalidad proporcionan (al menos a veces) formas de determinar las diferencias de fitness independientes del éxito reproductivo actual (Beatty 1980, Brandon \& Beatty 1984, Millstein en prensa).

Nuestra meta aquí es principalmente clasificar todas estas relaciones dividiéndolas en dos tipos. No realizar esta distinción podría llevar, y usualmente lleva, a mezclar planos diferentes (y en principio independientes) al discutir los modelos de optimalidad. En la sección 2, presentaremos dos formas en las que la selección natural es usada en la biología evolutiva: como una explicación histórica que da cuenta de la adaptación ${ }^{1}$ de los organismos al ambiente, un como una explicación ahistórica (en un sentido que se clarificará más adelante), que da cuenta de las diferencias en el éxito reproductivo entre organismos de distinto tipo en una población. En la sección 3 daremos un ejemplo de un modelo de optimalidad tomado del campo de la teoría del forrajeo óptimo (en adelante OFT). En las secciones 4 y 5 mostraremos las dos formas en que los modelos de optimalidad pueden utilizarse -en relación en ambos tipos de explicaciones selectivas-, mostrando como los problemas mencionados en esta sección pueden clasificarse como perteneciendo a uno u otro uso, e introduciremos algunas alternativas existentes y algunas nuevas a ellos. Finalmente, la última sección contiene algunas conclusiones.

\section{Dos tipos de explicaciones por selección natural}

En esta sección, siguiendo a Ginnobili $(2010,2014,2016)$ presentaremos dos formas en las que la selección natural es utilizada. A veces, los autores presentan a la selección natural como un proceso que involucra muchas generaciones, con el objetivo de explicar el origen de las adaptaciones, es decir, para explicar cómo ciertas poblaciones de organismos han adquirido rasgos altamente especializados, que les permiten sobrevivir y reproducirse de manera altamente efectiva en el ambiente en el que viven (Kitcher 1993, Skipper \& Millstein 2005). Un ejemplo de este modo de considerar a la selección natural, que asume esta posición, es el de Kitcher (1993, p. 28):

\section{Pregunta: ¿Por qué (prácticamente) todos los miembros de G tienen P?}

\footnotetext{
${ }^{1}$ El término adaptación también es usado de manera ambigua, como un rasgo que desarrolla cierta función de manera muy eficiente, o como un rasgo que ha sido moldeado por medio de procesos selectivos (Burian 1994, Ginnobili 2010, Gould \& Vrba 1982, WestEberhard 1994). En este artículo, cuando hablamos de "adaptaciones", nos referimos al primero de estos usos.
} 
Respuesta:

(1) Entre los ancestros de G había un grupo de organismos contemporáneos, $G_{0}$, tal que: (i) un número pequeño de miembros de G0 tenían p; (ii) ninguno de los miembros de la generación ancestral de $\mathrm{G}_{0}$ tenía $P$; (iii) cada uno de los otros miembros tienen una de las variantes características $P_{1}, \ldots, P_{n}$; (iv) ninguna otra variante de $P$ está presente en ninguna generación del linaje $G_{0}-G$

(2) Análisis de las condiciones ecológicas y efectos fisiológicos sobre los portadores de $P, P_{1}, \ldots, P_{n}$. que muestra que:

(3) Los organismos con $P$ tenían un mayor éxito reproductivo esperado que los organismos $P_{i}(1 \leq i \leq n)$.

(4) $P, P_{1}, \ldots, P_{n}$ son heredables

Por lo tanto

(5) $P$ incremento en frecuencia en cada generación del linaje de $G_{0}$ a $G$

(6) Hay suficientes generaciones entre Go y G.

Por lo tanto

(7) (prácticamente) todos los miembros de G ahora tienen $P$

Este tipo de presentación contrasta con otro en el cual la teoría de la selección natural es presentada a través de una ley o principio fundamental, que afirma que las diferencias en la posesión de un cierto rasgo llevan a diferencias en el éxito reproductivo de sus organismos poseedores (Brandon 1978, 1990, Endler 1986, Williams 1970). Por ejemplo, según Brandon, el "principio" de selección natural (PNS, como el mismo lo llama) tendría la siguiente forma:

[PNS]: Si a [tiene un mayor fitness] que b en el ambiente E entonces (probablemente) a tendrá más descendencia (suficientemente similar) que b en E. (Brandon 1978, p. 187, modificamos el antecedente para que se corresponda con nuestros usos terminológicos)

Esta presentación solo involucra a la transición entre dos generaciones, y tiene como explanandum, no la fijación de adaptaciones, sino algo menos mediato, las diferencias en el éxito reproductivo.

Llamaremos "histórica" a la primera forma de pensar la selección natural, en el sentido de que involucra muchas generaciones, y "ahistórica" a la segunda, en el sentido de que sólo considera dos puntos temporales, y que puede involucrar a sólo dos generaciones. ${ }^{2}$

La relación entre ellas puede verse en los ejemplos citados. Un principio ahistórico, como el de Brandon, tiene un rol central en la explicación histórica (véase los puntos 2 y 3 en patrón explicativo de Kitcher). Esto es, las explicaciones históricas contienen en su núcleo, entre otras cosas, a iteraciones del principio ahistórico; son las diferencias en el éxito reproductivo a lo largo de muchas generaciones las que explican la fijación del rasgo adaptativo. ${ }^{3}$ De este modo, tanto el explanandum como el explanans de las explicaciones selectivas ahistóricas son parte del explanans de las explicaciones adaptativas históricas. En consecuencia, cualquiera sea el rol que se adscriba a los modelos de optimalidad en relación con el explanans de la explicación ahistórica, lo cumplirá también en el explanans de la explicación histórica (dado que las explicaciones ahistóricas son parte de él).

Un ejemplo puede ayudar a clarificar esta distinción. Una cosa que Darwin se proponía explicar es el hecho de que las jirafas tienen largos cuellos que les permiten alimentarse de las ramas más altas de los árboles de acacias (una adaptación). La explicación de Darwin de este hecho apela, primero, a que las jirafas que tenían cuellos un poco más largos en una generación dada, tendían a ser más aptas -en este caso, sobrevivían más-, y en consecuencia tenían un mayor éxito reproductivo que las jirafas de cuellos más cortos (una explicación ahistórica de las diferencias en el éxito reproductivo entre diferentes jirafas).

\footnotetext{
2 A pesar de que estas explicaciones "a corto plazo" no son estrictamente ahistóricas, dado que involucran a al menos dos generaciones, elegimos esta terminología en lugar de "corto plazo" y "largo plazo" para notar que son distintos tipos de explicaciones (tienen diferentes explananda, una de ellas es predictiva mientras que la otra, discutiblemente, no lo es, etc.), y no meramente el mismo tipo de explicación, pero considerada en una mayor o menor escala temporal.

${ }^{3}$ Ginnobili (2014) presenta esta distinción a partir de la idea de que las explicaciones seleccionistas históricas y ahistóricas pueden pensarse a través de la distinción entre dos teorías, la teoría de la selección natural histórica y la teoría de la selección natural ahistórica, diferentes, aunque relacionadas. Uno de los puntos que dan plausibilidad a dicha opción reconstructiva es que las explicaciones seleccionistas históricas no están constituidas únicamente por iteraciones del de las explicaciones ahistóricas, sino que incluyen otros elementos, por ejemplo, la alusión a mecanismos de herencia y variación, de los que se prescinde en la explicación ahistórica. En este trabajo nos alcanza con sostener la tesis más débil, de que existen dos formas en las que la selección natural se utiliza a la hora de brindar explicaciones.
} 
Después de muchas generaciones, si sigue habiendo variación en el largo del cuello y si se mantienen las condiciones ambientales, este proceso que produce las diferencias en el éxito reproductivo se mantendrá (es decir, se iterará la explicación ahistórica), de modo que la población de jirafas adquirirá el rasgo en cuestión (Darwin 1872, pp. 177-178). ${ }^{4}$

Confusamente, tanto el proceso selectivo histórico como el ahistórico son llamados en la literatura "selección natural". Esta ambigüedad es responsable de varios malentendidos. Por ejemplo, de acuerdo a varios autores la teoría de la selección natural no es histórica (p.ej. Lloyd 1994), mientras que otros consideran a la selección natural como un dispositivo explicativo intrínsecamente histórico (p.e. Popper 1979, Smart 1963). Similarmente, ha habido disputas respecto al explanandum de la teoría de la selección natural (p.ej. Stegmann 2010, Stephens 2007, pp. 114-116). Con esta distinción en mente, estos problemas pueden deberse a que los autores tienen diferentes tipos de explicaciones seleccionistas en mente. En el contexto de este trabajo, como veremos en la sección 4, la distinción es relevante puesto que algunas objeciones tienen que ver con el rol que los modelos de optimalidad cumplen en las explicaciones históricas, mientras que otras con su rol en las ahistóricas.

\section{Modelos de optimalidad (un ejemplo)}

Por otra parte, suele decirse que los modelos de optimalidad contienen tres componentes o "partes". Estos consisten en (a) un rasgo tipo que puede asumir cierto rango de valores; $(b)$ una "moneda de cambio" (currency) o variable a ser optimizada; y (c) un conjunto de constricciones, que pueden ser de diferente tipo (físico/mecánicas, ambientales, del desarrollo, etc.). El ejemplo que presentamos aquí proviene del campo de la OFT, y fue propuesto por Schoener (1974). Este modelo estudia la dieta óptima que un predador debería adoptar (por esto debe entenderse los tipos de presa que debería incluir en ella). Así, el elemento (a), el rasgo tipo, es la dieta del predador, y sus posibles valores son todas las combinaciones diferentes de presas disponibles en el ambiente. Por ejemplo, si en un determinado territorio de caza hay tres tipos diferentes de presas (dígase, triángulos, círculos y cuadrados, notados como T, C y S), entonces las dietas posibles serán $\{T\},\{C\},\{S\},\{T, C\},\{T, S\},\{C, S\}$ y $\{\mathrm{T}, \mathrm{C}, \mathrm{S}\}$. Lo que Schoener eligió como criterio de optimización o currency es la eficiencia energética de cada dieta (medida como las calorías por unidad de tiempo obtenidas de ella en promedio). Las constricciones del modelo incluyen a algunas de tipo energético (como la energía promedio empleada en búsqueda, manipulación y digestión ${ }^{5}$ de las presas, y la energía obtenida de las presas incluidas en la dieta) y a otras basadas en el factor tiempo (tiempo empleado buscando y manipulando a presas en la dieta). Algunas de ellas, a su vez, pueden ser pensadas como nociones definidas; por ejemplo, el tiempo promedio empleado en búsqueda de presas depende del número de presas incluidas en la dieta (incluir a más ítems en la dieta disminuye el tiempo de búsqueda), la abundancia de tales presas, la velocidad a la que el predador se mueve o registra su ambiente, etc.

El trade-off siendo modelado aquí es el siguiente. Los ítems de presa pueden ser ordenados en un ranking de acuerdo a la energía neta que brindan (energía total que brindan menos la energía empleada en manipularlas) sobre el tiempo promedio empleado en su persecución. Es decir, la presa que se encuentra más alto en el ranking siempre debería ser incluida en la dieta ya que, una vez encontrada, devuelve la mayor cantidad de energía por el tiempo empleado en perseguirla. Incluir en la dieta sólo a presas que se encuentran alto en el ranking hará a energía promedio ganada por cada unidad de tiempo empleada en persecución muy alta. Sin embargo, si esas presas de alta calidad no son muy

\footnotetext{
${ }^{4}$ Con respecto a Darwin, también es interesante notar que estaba principalmente interesado en el origen de las adaptaciones, esto es, en la explicación de tipo histórica. Su objetivo principal era proveer una explicación de cómo los organismos se adaptan a sus ambientes que pudiera competir con aquella dada por los teólogos naturales (Darwin 1859, p. 3). Con el advenimiento de la genética de poblaciones, donde el explanandum consiste principalmente en los cambios (o las estabilidades) de las frecuencias de alelos y genotipos en una población, el interés cambia a la explicación ahistórica, dado que la selección natural ahistórica es uno de los mecanismos que pueden modificar las frecuencias esperadas.

5 Estas últimas tres son a veces abreviadas como "tiempo de manipulación”. En adelante adoptaremos este uso.
} 
abundantes, ello hará a los tiempos y costos energéticos empleados en búsqueda altos. Toda esta información puede representarse matemáticamente en la siguiente ecuación: ${ }^{6}$

$$
\frac{\mathrm{e}}{\mathrm{t}}(\mathrm{D})=\frac{\text { Promedio de energía neta de } \mathrm{D}-\text { Promedio de energía en búsqueda de } \mathrm{D}}{\text { Promedio de tiempo de persecución de } \mathrm{D}+\text { Promedio de tiempo de búsqueda de } \mathrm{D}}
$$

Por tanto, a un predador puede convenirle incluir presas de menor calidad en su dieta, reduciendo la ganancia energética promedio del tiempo empleado en persecución, pero incrementando la frecuencia con la que se encuentra con presas. Podemos concebir al predador como comenzando con una dieta que consiste sólo en la presa más alta en el ranking. Luego, va agregando, una por una, presas de menor calidad en su dieta, hasta que la disminución en el tiempo y energía empleados en búsqueda dejan de compensar por la disminución en la energía ganada por cada acto de caza y los incrementos en el tiempo promedio de persecución. A partir de aquella ecuación y este tipo de consideraciones, Schoener deriva un óptimo matemático. ${ }^{7}$

El modelo de Schoener incluye a una serie de asunciones que pueden no valer en situaciones reales. Por ejemplo, asume que el predador busca a todos los tipos de presa al mismo tiempo; también, que sólo la eficiencia energética importa y no, por ejemplo, los componentes nutricionales de la dieta, o la energía total obtenida (i.e. no hay un mínimo de energía sin el cual el predador no pueda sobrevivir, ni un máximo más allá del cual la energía no pueda ser utilizada); asume que el predador tiene a su disposición toda la información relevante acerca del ambiente, y no necesita muestrearlo antes de salir de caza; y que no hay riesgo para él al salir a cazar (él mismo no es presa de algún otro predador); etc. Estas asunciones pueden ser canceladas, formulando modelos más complejos (como sucede con el equilibrio Hardy-Weinberg en la genética de poblaciones). Por ejemplo, Krebs et al. (1977), y luego Krebs, Kacelnik y Taylor (1978) examinan lo que sucede cuando la asunción de información relevante es cancelada.

Finalmente, nos referimos al modo en el que estos modelos son evaluados o testeados. En algunos casos, ellos pueden estar "construidos" específicamente para representar a una especie particular que habita en un ambiente particular (p.ej. Holling 1964). Si el modelo representa adecuadamente a esos individuos (i.e. los rasgos que caracteriza como óptimos son de hecho encontrados), puede decirse que es "adecuado" o que ha sido "confirmado". Si no lo hace, que ha sido "refutado". Otras veces, como en el presente caso, los modelos pueden tener una aplicabilidad pretendida más amplia, pudiendo ser testeados en poblaciones de muchas especies diferentes. En este caso, el hecho de que una especie particular no se comporte del modo especificado por el modelo como óptima no necesariamente significa que el modelo debería ser descartado (ya que aun así podría representar adecuadamente a otras poblaciones o especies).

Sea cual sea su dominio de aplicaciones pretendidas, testear un modelo de optimalidad consiste, en primer lugar, determinar los parámetros involucrados, y los valores de currency para cada rasgo caso posible (en el modelo anterior, determinar los valores de $e / t$ para cada dieta particular); $y$, en segundo lugar, en chequear si la población real posee el rasgo que es óptimo de acuerdo al modelo (i.e. aquel con el valor de currency más alto).

Los modelos de optimalidad también pueden hacer algunas predicciones generales, las cuales pueden ser testeadas por separado. Por ejemplo, el modelo de Schoener considerado arriba predice que la inclusión de un nuevo tipo de individuo en la dieta no depende de su propia abundancia sino sólo de la abundancia de presas que se encuentras más arriba en el ranking; que la introducción de un competidor sólo puede ampliar la dieta, no restringirla, etc. La primera de estas predicciones también ha sido testeada por Krebs et al. (1977).

\footnotetext{
${ }^{6}$ Por simplicidad, está presentada aquí de modo semi-formal. Para la versión completa véase Schoener (1974), Roffé (2016, capítulo 4).

${ }^{7}$ Esto también tiene una formulación (y una derivación) matemática en el artículo de Schoener. No se la incluye aquí por no ser muy relevante para los propósitos de este artículo.
} 


\section{Los modelos de optimalidad y la selección natural histórica}

En esta sección y la siguiente, analizamos los usos que los modelos de optimalidad pueden tener en las explicaciones por selección natural. Comenzamos aquí con su lugar en las explicaciones seleccionistas históricas; en la sección siguiente nos centraremos en las ahistóricas. Nuestro objetivo es mostrar que el conjunto de los problemas y objeciones mencionados en la sección 1 (y otros) pertenecen a uno u otro uso.

Comenzamos notando un hecho obvio: que un rasgo sea óptimo (de acuerdo a algún modelo) es indicativo de que es una adaptación (en el sentido no evolutivo del término), y posteriormente, y más mediatamente, que pudo haber surgido por selección natural. Ahora, la inferencia desde el hecho de que un rasgo es óptimo respecto a algún currency a la conclusión de que el rasgo fue moldeado por la selección natural no es lógicamente necesaria, sino que es abductiva. En otras palabras, es una inferencia a la mejor explicación disponible, dado actualmente que la teoría de la selección natural brinda la mejor explicación de la adaptación (heredable). Sin embargo, hay (o al menos había) otras maneras alternativas de explicar este hecho.

De hecho, como se dijo en la introducción, los estudios de optimalidad (en general) nos son característicos sólo del marco darwiniano, ni siquiera del marco evolutivo. Por ejemplo, constituían una parte fundamental de la teología natural pre-darwiniana. A pesar de que los teólogos naturales eran conscientes de que los diseños en la naturaleza no son siempre óptimos (véase Blanco 2008), la búsqueda de la adaptación perfecta ocupaba un lugar central en sus estudios. Por ejemplo, Paley nota que la curva en el cristalino de los peces es óptima para compensar por la refracción de la luz en el agua (Paley 1809). Kirby, por su parte, nota que la forma y peso de las aletas de los peces sirve no sólo para la locomoción, sino que están óptimamente diseñadas para servir de contrapeso cuando el pez está quieto (Kirby 1836 vol. II, pp. 262-263). Estos diseños óptimos (así como otros sub-óptimos) constituyen el explanandum de la teoría del diseño inteligente de los teólogos naturales, así como de la teoría de la selección natural darwiniana. Como se dijo, lo que Darwin buscaba era una explicación alternativa de los mismos hechos. No tenerla habría puesto a las ideas transformistas en jaque (véase Darwin 1859, p. 3). En este sentido, los estudios de optimalidad pueden funcionar en la determinación del explanandum del diseño inteligente, de la selección natural, o de otros mecanismos que producen adaptaciones, como los de Lamarck. Que el diseño (más o menos) óptimo constituye el explanandum de todas estas teorías también es patente que Darwin mismo uso el hecho de que los diseños suelen ser sub-óptimos como un argumento en contra de la teología natural, y a favor de la selección natural (Darwin 1859, pp. 472-475). Es decir, a pesar de que los teólogos naturales eran conscientes de la imperfección de las adaptaciones, Darwin y muchos otros han considerado que la teoría de la selección natural brinda una mejor explicación de las adaptaciones imperfectas (Sober 2000). Pero este argumento sólo tiene sentido si los teólogos naturales también apuntaban a explicar la presencia de adaptaciones.

El punto relevante para nosotros es que el vínculo entre optimalidad y selección natural (histórica) puede no ser tan fuerte como a veces se asume que lo es -i.e. no es uno de implicación lógica, sino sólo de inferencia a la mejor explicación-. Este punto también es relevante para las discusiones acerca del carácter supuestamente adaptacionista de los modelos de optimalidad. La tesis de que todo rasgo en la naturaleza es óptimo -llámese a esta tesis "optimalismo”, una tesis que Paley podría haber compartidono implica lógicamente que la presencia de todo rasgo debería ser explicada por medio de procesos selectivos, con la exclusión de todo otro posible mecanismo alternativo (una de las objeciones que Gould \& Lewontin 1979, hacen al programa adaptacionista). En otras palabras, el adaptacionismo -o esta versión de él- se obtiene asumiendo dos cosas: optimalismo, más la asunción de que la selección es la única explicación posible de los diseños óptimos en la naturaleza.

Nótese también que si la segunda parte se asume como verdadera, entonces el optimalismo es lo que implica que si un modelo falla en predecir los rasgos reales, entonces otro necesita ser propuesto 
para dar cuenta de su presencia (las acusaciones de infalsabilidad mencionadas en la sección 1 a menudo apuntan a esto). Sin embargo, si se acepta que no todo rasgo en la naturaleza es óptimo (ni siquiera de un modo constreñido), entonces posiciones más débiles se vuelven posibles. Por ejemplo, la posición de Potochnik (2009), en la cual se sostiene que un modelo de optimalidad puede indicar únicamente los valores que el rasgo tendría si hubiese estado sujeto sólo a la selección, y que permite sacar como conclusión "el rasgo simplemente no es óptimo" (dado que otros factores intervinieron) de la falla de un modelo, en lugar de la conclusión "otro modelo necesita ser propuesto".

Por supuesto, los modelos considerados en sí mismos no implican ninguna de estas posiciones. No hay nada, por ejemplo, en el modelo de Schoener, que afirme que toda dieta de todo predador posible deba conformarse a las ecuaciones presentadas. La pregunta interesante concierne a las intenciones y el comportamiento de las comunidades relevantes. Nuestra meta aquí no es tomar posición respecto de la verdad o falsedad del optimalismo, del adaptacionismo, etc. ni acerca de su utilidad como heurísticas, sino sólo mostrar el modo como los modelos de optimalidad, y el rol que juegan en el explanandum de las explicaciones seleccionistas históricas, se relacionan con estas tesis. Más específicamente, hemos apuntado a mostrar que ellos no implican lógicamente al adaptacionismo, al menos no por sí mismos, y que otras tesis auxiliares (como el optimalismo) son necesarias para obtenerlo.

Otro problema relacionado es la objeción (también hecha por Gould \& Lewontin 1979) a la inferencia que va desde la perfección de una adaptación (i.e. el hecho de que un diseño dado es óptimo) para realizar cierto comportamiento o función, a la conclusión de que evolucionó por selección natural para hacer lo que (óptimamente) hace actualmente (véase Ginnobili \& Blanco 2007). Puede notarse que modelos como el presentado en la sección 3 son sincrónicos, y no necesariamente brindan conocimiento acerca de la historia del rasgo. Es decir, incluso si la práctica del modelado de optimalidad implicara al adaptacionismo, en el sentido de que nos llevara a creer que la presencia de todo rasgo debe ser explicada por medio de explicaciones seleccionistas históricas, los modelos de optimalidad en sí mismos podrían no decirnos nada acerca de la historia de esos rasgos, dado que estos podrían ser "exaptaciones” (Gould \& Vrba 1982).

Finalmente, un último problema concerniente a este tipo de uso es la idea de Collin Rice, de que los modelos de optimalidad brindan explicaciones por equilibrio de los estados a los que la selección natural llevará a las poblaciones (Rice 2012, pp. 686-687). Es posible ver que por "selección natural", Rice se está refiriendo aquí a la selección natural histórica -i.e. los equilibrios en cuestión no son equilibrios genotípicos como los de la genética de poblaciones, sino equilibrios en el sentido de estados adaptativos en los que un rasgo se encontrará fijado-. De este modo, él cree que los modelos de optimalidad permiten no sólo especificar el explanandum de las explicaciones históricas, sino también su predicción. Es decir, en la concepción de Rice, no sólo sería el caso que si encontrásemos un predador cuya dieta es óptima, entonces deberíamos tratar de explicarla intentando dar con una explicación en términos histórico-seleccionistas. También sería el caso de que sea cual sea la dieta actual que encontremos en el predador, podríamos entonces predecir cuál será su dieta, en alguna generación remota, construyendo un modelo de optimalidad (y asumiendo que las asunciones que el modelo hace, de acuerdo a Rice, se cumplen). Por lo tanto, no es conceptualmente necesario que todo uso de un modelo de optimalidad en el explanandum de explicaciones históricas se refiera a la acción pasada de la selección natural (aunque la tesis de Rice podría ser, también, falsa).

\section{Los modelos de optimalidad y la selección natural ahistórica}

En esta sección, analizamos el otro rol (o conjunto de roles) que los modelos de optimalidad pueden jugar en relación con la selección natural, aquellos pertenecientes a su relación con el explanans de la teoría de la selección natural ahistórica. Este debate concierne especialmente a la habilidad de los modelos de optimalidad de determinar las diferencias de fitness. 
Una primera posición, algo extrema, niega que los modelos de optimalidad puedan jugar algún rol en determinar las diferencias de fitness dentro de las explicaciones seleccionistas ahistóricas. Esta parece ser la posición tomada por autores como Bolduc y Cézilly (2012). Ellos afirman que:

\begin{abstract}
La teoría del forrajeo óptimo, no permite realizar ninguna afirmación acerca de la preeminencia de la selección natural en la evolución pasada de los rasgos, ni del moldeado actual de rasgos por la selección natural. [...] En el campo de la ecología del comportamiento, OFT es simplemente usada como una herramienta para investigar los factores actuales (parámetros y variables que caracterizan a un organismo o a algún factor ambiental) que están causalmente conectados con el comportamiento de los individuos (Bolduc \& Cézilly 2012, pp. 853-854).
\end{abstract}

La primera oración parece estar negando la capacidad de los modelos de OFT de ser usados dentro de ambas explicaciones seleccionistas, histórica y ahistórica. Sin embargo, si se observa más detalladamente a la segunda oración, parece haber un rol en el caso histórico, aunque uno débil: la especificación de los parámetros relevantes del ambiente (nótese que el ambiente está presente en la formulación del PNS que hemos visto) -en esto parecen estar siguiendo a Maynard Smith (1978)-. Bajo esta perspectiva, cualquier intento de determinar las diferencias de fitness a partir de modelos de optimalidad sería inadecuada, y por eso la llamamos a la posición "extrema".

Otras posiciones - menos extremas- también pueden encontrarse en la literatura. Una manera general de introducirlas es señalando lo siguiente. Como se dijo, que un rasgo presente en una población esté óptimamente diseñado para resolver algún problema ambiental es lo que Darwin quería explicar con la teoría de la selección natural; pero al mismo tiempo, la historia adaptativa que propone para explicar la adquisición de este rasgo parece apelar al hecho de que, en el pasado, variantes cuyos rasgos estaban más cerca de los valores óptimos tenían su éxito reproductivo diferencial incrementado, respecto de las variantes cuyos rasgos eran menos óptimos. Así, las jirafas cuyos cuellos se aproximaban más al largo óptimo para comer de las ramas altas de las acacias eran las que sobrevivían más, y por tanto tenían un mayor éxito reproductivo.

Usualmente se asume que el PNS tiene principalmente dos "partes" o dos componentes conceptuales centrales: fitness y éxito reproductivo. Por ejemplo, como se mostró en la sección 2, la formulación de Brandon del PNS mantiene que si $a$ tiene un mayor fitness que $b$ en el ambiente $E$ entonces (probablemente) a tendrá más descendencia (suficientemente similar) que $b$ en E. Por lo tanto, bajo esta concepción del PNS, si los modelos de optimalidad fuesen a desempeñar algún rol en el explanans de las explicaciones seleccionistas ahistóricas (como las posiciones que ahora estamos considerando lo hacen) solamente permitirían hacerlo permitiéndonos determinar las diferencias de fitness entre distintos tipos de individuos (descartando la idea de que nos permiten determinar E). Ese es precisamente el rol que la interpretación propensionista del fitness le adscribe a los modelos de optimalidad (Beatty 1980, Brandon \& Beatty 1984). Por ejemplo, Roberta Millstein lo puso de este modo recientemente:

Con la interpretación propensionista, si buscamos explicar por qué el tipo A está dejando un mayor número de descendientes que el tipo B, la explicación "porque A tiene un mayor fitness que B" significa que $\mathrm{A}$ tiene una mayor propensión que $\mathrm{B}$ a sobrevivir y reproducirse en el ambiente dado, lo cual significa que las propiedades físicas de $\mathrm{A}$ en su ambiente son lo que causan que tienda a tener un mayor éxito reproductivo que B (con sus propiedades fisicas). Las habilidades fisicas relativas pueden determinarse por medio de modelos de optimalidad ingenieriles $\mathrm{u}$ otros modos de examinar las propiedades físicas de los organismos en su ambiente, y luego ser confirmadas por mediciones de la contribución real a la descendencia (Millstein en prensa, énfasis nuestro).

Este enfoque tiene una consecuencia ligeramente extraña, ya que el PNS tendría un status peculiar: sería una parte de la teoría de la probabilidad (Brandon 1978, 2006, p. 333). Esto es, de acuerdo a los propensivistas, el concepto de fitness debería ser entendido como la probabilidad de dejar cierto número de descendientes, o de tener cierta medida de éxito reproductivo (dado que las propensiones son modos de interpretar a conceptos probabilísticos). Por lo tanto, el PNS (en la formulación de Brandon) establecería meramente que los individuos que probablemente tendrán un mayor éxito 
reproductivo que otros, tenderán, de hecho, a tener un mayor éxito reproductivo que esos otros. En otras palabras, que las disposiciones probabilísticas tenderán a manifestarse en casos reales, lo cual no parece una afirmación genuinamente empírica.

El modo preciso de cómo estas determinaciones del fitness ocurrirían es también debatido, habiendo diferentes propuestas en la literatura. Por ejemplo, Beatty (1980) afirma que los valores de currency provenientes de modelos de optimalidad podrían funcionar como "sustitutos" de los valores de fitness, mientras que Brandon (1978) ve a los modelos "ecológicos" (de optimalidad) como modos de instanciar el concepto de fitness (de ese modo volviendo al PNS, que es un esquema de ley general, una afirmación empírica aplicable pero menos general).

Una tercera posición posible acerca de la relación entre optimalidad y explicaciones seleccionistas ahistóricas -en las que el PNS no tiene el mencionado estatus peculiar, manteniendo su carácter de ley fáctica tradicional- puede introducirse como sigue. Algunos autores (p.ej. Casanueva 2011, Ginnobili 2010, 2016) ven al PNS como teniendo una estructura más compleja. La línea de razonamiento que siguen para alcanzar esta conclusión poco ortodoxa es algo como lo siguiente. Si uno ve una explicación darwiniana típica del éxito reproductivo diferencial, es posible encontrar otros conceptos (además del fitness y el éxito reproductivo) jugando roles en el explanans. Considérense, por ejemplo, a los siguientes casos:

[Entre las jirafas] los individuos que eran los pacedores más altos y eran capaces, durante las sequías, de alcanzar incluso una pulgada o dos por sobre los otros, habrán sido preservados más frecuentemente [...] aquellos individuos que tenían alguna parte o varias de sus cuerpos más alargadas que lo usual, generalmente habrían sobrevivido. Estas se habrían cruzado y dejado descendencia, la cual heredaría las mismas peculiaridades corporales, o una tendencia a variar del mismo modo; mientras que los individuos menos favorecidos en los mismos aspectos habrían sido más pasibles de perecer (Darwin 1872, pp. 177-178).

Aquellas flores individuales que tenían las glándulas o nectarios más grandes, y que excretaban más néctar, serían más frecuentemente visitadas por insectos, y serían más frecuentemente cruzadas; así, en el largo plazo, llevarían la ventaja (Darwin 1859, p. 92).

Lo que ambas explicaciones tienen en común es que contienen al menos tres "partes" diferentes: (i) Jirafas que son mejores para alimentarse/Plantas con flores que son más visitadas por insectos, (ii) Jirafas que sobreviven más que otras/plantas que son más fecundas que otras, (iii) Jirafas y plantas que tienen un mayor éxito reproductivo que aquellas otras. La práctica biológica más reciente conserva esta explicación en tres partes. Considérese, por ejemplo, el caso famoso de las polillas Biston betularia, como recientemente era enseñado en manuales de biología evolutiva actuales. Ridley (1996) lo presenta del siguiente modo:

Podemos ilustrar el proceso con un ejemplo famoso [...] La forma usual de la polilla moteada Biston betularia en el norte de Europa tiene un patrón de coloración "moteado" y claro [...]. La polilla descansa sobre las ramas de los árboles, y su patrón de color la camufla contra ataques de predadores. Sin embargo, el camuflaje funciona sólo contra el fondo adecuado -ramas con coloración clara, la cual es causada principalmente por el crecimiento de líquenes-. Durante la revolución industrial en el Reino Unido, la polución del humo mató a los líquenes cerca de las áreas industriales, dejando a las ramas de los árboles negras. Aproximadamente en este momento (alrededor de 1830), una forma "melánica" de la polilla se volvió incrementalmente común en las colecciones de polillas contemporáneas; esta forma melánica estaba camuflada en las ramas oscuras de los árboles. A lo largo del siglo 19, la forma melánica aumentó en frecuencia hasta que, cerca de las regiones industriales, fue reconocida como el tipo normal de polilla.

El incremento de la forma melánica de esta polilla estuvo casi ciertamente impulsado por la selección natural. Observaciones hechas por Kettlewell en el siglo veinte revelaron que era más probable que los pájaros comieran a polillas pobremente camufladas. Adicionalmente, cuando ambas formas fueron liberadas en áreas industriales era más probable la recaptura posterior de la melánica. Kettlewell también notó que cuando eran liberadas en áreas no industriales, era más probable recapturar a la forma moteada (Ridley 1996, pp. 72-73). 
Como el lector puede notar, esta es una explicación de tipo histórico, en el cual una adaptación (la posesión de un color de alas que camufla a las polillas a los ojos de sus predadores) es adquirida por medio de la repetición o la iteración de un proceso ahistórico. Según la presentación de Brandon del PNS (véase más arriba), la instancia del proceso ahistórico en juego aquí tendría los siguientes elementos:

Si las polillas que tienen alas de color más oscuros tienen un mayor fitness (tienden a sobrevivir más) que las de alas claras, en un ambiente $\mathrm{E}$ (en el que la polución mató a los líquenes, y las ramas de los árboles donde las polillas descansan se volvieron oscuras, etc.), entonces las de color más oscuro tendrán (probablemente) un mayor éxito reproductivo que las de color más claro.

Puesto de modo semi-formal, y más gráfico:

Las polillas con el color a sobreviven más que las polillas con el color de alas $\mathrm{b}$ en el ambiente $\mathrm{E} \rightarrow$ Las polillas con el color de alas a tienen un mayor éxito reproductivo que las polillas con el color de alas $\mathrm{b}$

Una de las cosas que usualmente se piensa que hizo Kettlewell es la de medir precisamente las diferencias entre las frecuencias de supervivencia de las polillas, esto es, de medir cuantitativamente las diferencias de fitness, por medio del uso de los experimentos marcar-recapturar que menciona Ridley (Kettlewell 1955, 1956, véase también Ridley 2004, pp. 111-112). Es decir, lo que Kettlewell habría hecho en esos artículos es determinar el antecedente del condicional en esa instancia del PNS. Sin embargo, eso no es todo lo que Kettlewell hizo en ellos, y esos no son los únicos componentes en la explicación de la Biston betularia. Una parte central está ausente: el hecho de que las polillas oscuras están mejor camufladas en ese ambiente que las claras. Esta no es simplemente otra parte de la descripción del ambiente en el que el proceso selectivo está siendo llevado a cabo. Efectivamente, las diferencias en el camuflaje explican las diferencias en la supervivencia, ${ }^{8}$ con lo cual sería mejor pensarlos como otro antecedente en esa instancia del PNS:

Las polillas con el color a están mejor camufladas que las polillas con el color de alas b en el ambiente $\mathrm{E}$ Las polillas con el color a sobreviven más que las polillas con el color de alas b en el ambiente $\mathrm{E}$ Las polillas con el color de alas a tienen un mayor éxito reproductivo que las polillas con el color de alas $\mathrm{b}$

Lo que Kettlewell hizo en esos artículos no fue solamente determinar cuantitativamente el término en el consecuente del primer condicional de esta oración (i.e. la supervivencia diferencial de las polillas); también dedicó mucho tiempo a probar que el primer condicional es cierto. Esto es, realizó experimentos para mostrar que las diferencias en la supervivencia se debian a diferencias en la conspicuidad de las polillas. Con lo cual, incluso a pesar de que (a diferencia de Darwin) Kettlewell tenía acceso a mediciones cuantitativas de fitness, sin esta otra información, su explicación hubiese quedado incompleta (Roffé 2016). Ninguna de estas tres partes es vacua, ni puede ser eliminada sin pérdida. El patrón explicativo de las explicaciones seleccionistas ahistóricas tendría entonces todos estos componentes señalados.

Adicionalmente, si se supone que el PNS captura este patrón -como la ley o esquema de ley unificadora que todas las explicaciones seleccionistas ahistóricas comparten-, entonces debe ser reformulado para dar cuenta de estos conceptos faltantes, de aproximadamente el siguiente modo tripartito:

Los organismos con el rasgo a desarrollan un efecto o una función de modo más efectivo que los organismos con el rasgo b en el ambiente $\mathrm{E}$

Los organismos con el rasgo a tiene un mayor fitness que los organismos con el rasgo b en $\mathrm{E}$

Los organismos con el rasgo a tendrán un mayor éxito reproductivo que los organismos con el rasgo b. (adaptado de Ginnobili 2010, 2016)

\footnotetext{
${ }^{8}$ Algunos incluso dirían "causa". No entraremos en esta discusión, dado que nos llevaría lejos de nuestra meta.
} 
Con esta concepción del PNS en mente, sería posible adscribir a los modelos de optimalidad otro rol diferente en el explanans de las explicaciones seleccionistas ahistóricas. Efectivamente, ahora se vuelve posible afirmar que lo que nos ayudan a determinar directamente, al menos en algunas instancias (como aquellas tomadas de OFT), es el antecedente del primer condicional. Esto es, lo que algunos modelos de optimalidad nos podrían ayudar a determinar es el grado de efectividad con el que un rasgo desarrolla una cierta función biológica. El modelo de Schoener puede ser visto como una instancia de esto, ya que la eficiencia energética no es usualmente pensada como una instancia o componente del concepto de fitness. También podría haber problemas en pensarla como un "sustituto" del fitness, ya que el fitness y la eficiencia energética podrían no correlacionar linealmente (i.e. diferencias en una variable podrían no corresponder a iguales diferencias en la otra), y aun así el modelo ser considerado adecuado (porque los rasgos reales son predichos correctamente de él). Por otro lado, las diferencias en la eficiencia energética (o, de modo más general, en la habilidad para alimentarse) parecen explicar las diferencias en la supervivencia, motivo por el cual no serían simplemente una especificación del ambiente (como Bolduc y Cézily mantendrían). Por tanto, parece más natural pensar a un modelo como el de Schoener como un modo de determinar la eficacia con la que la función biológica de la alimentación es desarrollada.

Por supuesto, si Ginnobili $(2010,2016)$ tienen la razón, entonces determinar las diferencias en la eficacia con la que una función es desarrollada también nos permitiría determinar las diferencias de fitness, a través de la aplicación del PNS (así como uno puede determinar las aceleraciones a partir de fuerzas y masas, y la aplicación de la segunda ley de Newton). Pero el punto sería que esto sólo sería un modo indirecto de determinar a las diferencias de fitness, y por lo tanto la historia propensivista (la cual toma a esta relación como una más directa) sería una caracterización metateórica inadecuada de esta relación -al menos en lo que concierne a OFT-.

Nuevamente, nuestro objetivo aquí no es tomar posición en este debate, ${ }^{9}$ sino mostrar la variedad de opciones en las que los modelos de optimalidad podrían jugar roles en las explicaciones seleccionistas ahistóricas. Nótese también que el problema de la relación entre optimalidad (o valores de currency) y valores de fitness parece ser en alguna medida independiente los problemas considerados en la sección anterior. Incluso si los modelos de optimalidad sí nos permiten determinar u operacionalizar el fitness (del modo que sea, directamente o indirectamente a través del PNS), entonces pueden decirnos qué tipos de organismos tendrán un mayor éxito reproductivo en el corto plazo, pero no necesariamente cuál será el estado de la población o cuáles rasgos se fijarán en alguna generación remota, ni cuál era ese estado en alguna generación pasada. Si los modelos de optimalidad sólo cumpliesen este rol, entonces las acusaciones de presuponer el adaptacionismo (o incluso el optimalismo) parecerían fuera de lugar. Nada en esta posición involucraría que todos los rasgos son óptimos, ni que la selección natural ha moldeado todos los rasgos para estar óptimamente diseñados.

\section{Conclusión}

En este artículo hemos mostrado dos usos principales (o conjuntos de usos) que los modelos de optimalidad pueden tener, en relación con la teoría y las explicaciones de la selección natural. El concepto de optimalidad parece jugar dos roles distintos en la teoría evolutiva. En primer lugar, parece funcionar para determinar los explananda de las explicaciones seleccionistas históricas (para caracterizar aquello a lo cual puede darse este tipo de explicación seleccionista); y segundo, parece funcionar dentro del explanans de esas explicaciones, ya que explica por qué ciertos organismos tienen un mayor éxito que otros (i.e. porque sus rasgos se acercan más a los valores óptimos). Estos dos roles son, en principio, independientes entre sí y, como hemos visto, tienen diferentes conjuntos de problemas asociados con ellos. Esta distinción permite clarificar más adecuadamente la naturaleza precisa de tales problemas y sus relaciones mutuas, brindando algún orden a un panorama conceptual algo enrevesado. La

\footnotetext{
${ }^{9}$ A pesar de que el lector probablemente puede ver en donde recaen nuestras simpatías. Sí tomaremos posición y argumentaremos a favor de la tercera posición en un artículo que está bajo elaboración.
} 
discusión metateórica respecto a la naturaleza de los modelos de optimalidad y su rol en la biología, en este sentido, contribuye con la discusión respecto a su valor.

Bibliografía

Beatty, J. (1980), "Optimal-Design Models and the Strategy of Model Building in Evolutionary Biology", Philosophy of Science 47(4): 532-561.

Blanco, D. (2008), "La naturaleza de las adaptaciones en la teología natural británica: análisis historiográfico y consecuencias metateóricas”, Ludus Vitalis 16(30): 3-26.

Bolduc, J.S. y F. Cézilly (2012), "Optimality Modelling in the Real World", Biology $\mathcal{E}$ Philosophy 27(6): 851-869.

Brandon, R. (1978), "Adaptation and Evolutionary Theory", Studies in History and Philosophy of Science 9: 181-206.

Brandon, R. (1990), Adaptation and Environment, Princeton, New Yersey: Princeton University Press.

Brandon, R. y J. Beatty (1984), "The Propensity Interpretation of 'Fitness'-No interpretation Is No Substitute", Philosophy of Science 51(2): 342-347.

Brandon, R. (2006), “The Principle of Drift: Biology's First Law”, The Journal of Philosophy 103(7): 319-335.

Burian, R.M. (1994), “Adaptation: Historical Perspectives", en Keller, E.F. y E.A. Lloyd (eds.), Keywords in Evolutionary Biology, Cambridge: Harvard University Press, pp. 7-12.

Casanueva, M. (2011), "A Structuralist Reconstruction of the Mechanism of Natural Selection in Set Theory and Graph Formats”, en Martinez Contreras, J. y A. Ponce de León (eds.), Darwin's Evolving Legacy, México: Siglo XXI, pp. 177-192.

Darwin, C. (1859), On the Origin of Species by Means of Natural Selection, London: John Murray.

Darwin, C. (1872), On the Origin of Species by Means of Natural Selection, $6^{\text {th }}$ edition, London: John Murray.

Endler, J.A. (1986), Natural Selection in the Wild, Princeton, New Jersey: Princeton University Press.

Ginnobili, S. (2010), "La teoría de la selección natural darwiniana", Theoria 25(1): 37-58.

Ginnobili, S. (2014), "Explicaciones seleccionistas históricas y ahistóricas”, Ludus Vitalis, 33: 21-41.

Ginnobili, S. y D. Blanco (2007), "Gould y Lewontin contra el programa adaptacionista: elucidación de críticas", Scientiae Studia 5(1): 35-48.

Ginnobili, S. (2016), "Missing Concepts in Natural Selection Theory Reconstructions", History and Philosophy of the Life Sciences 38(8): 2-33.

Gould, S.J. y R.C. Lewontin (1979), "The Spandrels of San Marco and the Panglossian Paradigm: A Critique of the Adaptationist Programme", Proceedings of the Royal Society of London 205: 581-598.

Gould, S.J. y E. Vrba (1982), “Exaptation-A Missing Term in the Science of Form”, Paleobiology 8(1): 4-15.

Gray, R.D. (1987), "Faith and Foraging: A Critique of the "Paradigm Argument from Design.”, en Kamil, A.C., Krebs, J.R. y H.R. Pulliam (eds.), Foraging Behavior, New York: Springer US, pp. 69-140.

Henry, D. (2013), “Optimality and Teleology in Aristotle's Natural Science”, en Inwood, B. (ed.), Oxford Studies in Ancient Philosophy, vol. XLV, pp. 225-263.

Holling, C.S. (1964), "The Analysis of Complex Population Processes", The Canadian Entomologist 96(1-2): 335-347.

Kettlewell, H.B.D. (1955), "Selection Experiments on Industrial Melanism in the Lepidoptera”, Heredity 9: 323-342.

Kettlewell, H.B.D. (1956), "Further Selection Experiments on Industrial Melanism in the Lepidoptera”, Heredity 10(3): 287-301.

Kitcher, P. (1993), "Function and Design”, en Kitcher, P. (ed.), In Mendel's Mirror: Philosophical Reflections on Biology, Oxford: Oxford University Press, pp. 159-176.

Kirby, W. (1836), On the Power, Wisdom and Goodness of God, as Manifested in the Creation of Animals, and in their History, Habits and Instincts, Philadelphia: Carey, Lea \& Blanchard. 
Krebs, J., Erichsen, J., Webber, M. y E. Charnov (1977), "Optimal Prey Selection in the Great Tit (Parus major)", Animal Behaviour 25(1): 30-38.

Krebs, J.R., Kacelnik, A. y P. Taylor (1978), “Test of Optimal Sampling by Foraging Great Tits”, Nature 275(5675): 27. 31.

Lloyd, E.A. (1994), The Structure and Confirmation of Evolutionary Theory, New Jersey: Princeton University Press.

Millstein, R.L. (en prensa), "Probability in Biology: The Case of Fitness", en Hájek, A. y C.R. Hitchcock (eds.), The Oxford Handbook of Probability and Philosophy, Oxford: Oxford University Press. Accesible en: http://philsciarchive.pitt.edu/10901/.

Paley, W. (1809), Natural Theology, $12^{\text {th }}$ edition, London: J. Faulder.

Pierce, G.J. y J.G. Ollason (1987), "Eight Reasons Why Optimal Foraging Theory Is a Complete Waste of Time", Oikos 49(1): 111-118.

Popper, K. (1979), Objective Knowledge: An Evolutionary Approach, $2^{\text {nd }}$ edition, Oxford: Clarendon.

Potochnik, A. (2009), "Optimality Modeling in a Suboptimal World”, Biology $\mathcal{E}$ Philosophy 24(2): 183-197.

Rice, C. (2012), "Optimality Explanations: A Plea for an Alternative Approach", Biology E⿱ Philosophy 27(5): 685-703.

Ridley, M. (1996), Evolution, $2^{\text {nd }}$ edition, Cambridge, MA: Blackwell Science.

Ridley, M. (2004), Evolution, $3^{\text {rd }}$ edition, Malden: Blackwell.

Roffé, A. (2016), La genética de poblaciones y las fuerzas evolutivas, Tesis de licenciatura, Buenos Aires: Universidad de Buenos Aires.

Schoener, T.W. (1974), "The Compression Hypothesis and Temporal Resource Partitioning", Proceedings of the National Academy of Sciences of the United States of America 71(10): 4169-4172.

Skipper, R.A. y R.L. Millstein (2005), "Thinking About Evolutionary Mechanisms: Natural Selection”, Studies in History and Philosophy of Biological and Biomedical Sciences 36: 237-347.

Smart, J.J.C. (1963), Philosophy and Scientific Realism, London: Routledge and Kegan Paul.

Smith, J.M. (1978), "Optimization Theory in Evolution”, Annual Review of Ecology and Systematics 9: 31-56.

Sober, E. (2000), Philosophy of Biology, $2^{\text {nd }}$ edition, Boulder, Colorado: Westview Press.

Stegmann, U.E. (2010), "What can Natural Selection Explain?", Studies in History and Philosophy of Biological and Biomedical Sciences 41: 61-66.

Stephens, C. (2007), "Natural Selection”, en Matthen, M. y C. Stephens (eds.), Philosophy of Biology, The Netherlands: Elsevier, pp. 111-127.

West-Eberhard, M.J. (1994): "Adaptation: Current Usages", en Keller, E.F. y E.A. Lloyd (eds.), Keywords in Evolutionary Biology, Cambridge: Harvard University Press, pp. 13-8.

Williams, M.B. (1970), "Deducing the Consequences of Evolution: A Mathematical Model", Journal of Theoretical Biology 29(3): 343-385. 